\title{
Reviews for "Personality Trait Descriptors: 2,818 Trait Descriptive Adjectives characterized by familiarity, frequency of use, and prior use in psycholexical research"
}

\author{
Dear Drs Condon, Coughlin and Weston,
}

Thanks for submitting your work to JOPD for consideration. I really enjoyed reading more about your work, and as you may find below, the reviewers also had much to praise about your work. After review, we have reached a decision regarding your submission to Journal of Open Psychology Data, "Personality Trait Descriptors: 2,818 Trait Descriptive Adjectives characterized by familiarity, frequency of use, and prior use in psycholexical research". Our decision is to request (quite minor) revisions of the manuscript prior to acceptance for publication.

The full review information is included at the bottom of this email. Please note that there may also be a copy of the manuscript file with reviewer comments available once you have accessed the submission account. We ask you to please consider the following issues and revise the file accordingly.

There are not many changes to make, but I would like to offer the opportunity to make some revisions based upon the comments below. Due to their nature, provided that a comprehensive response is provided (and associated actions take place where you deem appropriate), I do not consider it necessary to require another round of review and will endeavour to action the resubmission as promptly as I can manage.

Instructions for how to resubmit your article online are pasted below. Please ensure that your revised files adhere to our author guidelines, and that the files are fully proofed prior to upload. Please also include a revised version of your article with 'tracked changes', adding comments where appropriate, to indicate the revisions made, in addition to a brief document outlining how you have responded to the reviewers' requests.

Please also ensure that all copyright permissions have been attained for any figures/tables you have included.

If you have any questions or difficulties during this process, please do contact us.

Please could you have the revisions submitted with four weeks. If you cannot make this deadline, please let us know as early as possible.

Thanks again for submitting such interesting and important work, we're grateful you considered JOPD, and we hope you enjoy and find it of benefit to reflect upon the feedback provided. Thanks, and take care,

Kind regards,

Dr Thomas Rhys Evans

University of Greenwich

Thomas.Evans@greenwich.ac.uk 
Reviewer A:

While I do have a couple of comments, I think this manuscript should be published. No additional rounds of reviews should be needed, but I'm willing to review a revision if the editor begs to differ.

(1). I've run parts of your R code and was able to read in the data and produce my own tables with minimal fuss, so well done. The $\mathrm{R}$ code is also written clearly.

(2). p. 3, penultimate paragraph: "aggregating of a trait descriptive adjective set" > "aggregating a ...".

(3). p. 6, penultimate paragraph: The text of the multiple-choice questions weren't made available. "This was done to maintain their validity for subsequent research." I don't understand this reason, so perhaps you could try to express yourselves more clearly here. I've prefixed this with an asterisk because one strength of your study is that each target word appeared in two line-ups rather than just one, but users of your data are unable to work out why the word 'direct' received correct responses by only $17 \%$ of participants in set A but by fully $97 \%$ in set $B$. (This is the item for which the discrepancy is most pronounced; about 2/3s of items have a less than 10 percentage-point discrepancy between both sets.)

(4). p. 7, first paragraph: "The extent to which the meaning was known was affected by both knowledge about the term-definition pair and agreement among respondents that the term was the best match for the definition given." I think I get what you mean, but I found this sentence confusing and I think it may be better to just delete it and adjust the next sentence accordingly: "Terms with higher proportions of correct responses [no scare quotes necessary] can be considered [rather than just "are"] to be more familiar than terms with lower proportions of correct responses." I don't think you can make any claims about ambiguity, because you only pitted each term against ten other terms, which were selected so as not to be closely related in meaning. It's perfectly possible for an item to be pretty ambiguous, but with respect to meanings that weren't relevant in the match-up.

(5). I think you can leave out the graphs, since they're also available on the website. Another minor comment about the graphs: I'd stick a "coord_flip()" at the end of the code for generating the bar plots, so that it's easier to read the labels along the x-axis.

(6). The fact that many participants had some higher education degree seems to have been an accident. Is there any way for users to filter out participants based on their level of education? (Or based on their region of residence, age etc.)

(7). There were a handful of words that appeared in forms C and D (e.g., close-fisted, hard-shelled, saturnine etc.). These weren't excluded from the tables in the interface, but I wasn't able to figure out where these words came from.

Jan Vanhove, November 6, 2021 
Reviewer B:

Thank you for inviting me to review the collection of this dataset on familiarity with personality trait descriptors. I believe that peer review should be an open and improvement-focused process, so I write my comments positively with the intention of helping improve the work. I will also sign my review below.

This submission is a much-needed and well-presented project. There has been a sore need to examine the familiarity/difficulty of the words used in common trait measures for some time. Common language has changed and advanced so much in the last $\sim 40$ years and a project like this is exactly is vital to understanding how valid standardised tools remain.

I find myself with no outstanding comments and recommendations for the text. Wherever I had questions as a reader (such as with the unexpected number of respondents with college degrees despite exclusion criteria) the authors addressed this in the text in a clear and transparent way. I also really appreciate the authors' clarity about the limitations on generalisability of the data outside of American English.

I recognise that the authors have a large sample size and I support the authors' use of a planned missing design. I would suggest that at some point in the sample section it would be useful to explain why this sample size was chosen (even if simply convenience of available funds) and why the $\mathrm{N}$ per term is sufficient. Can the authors declare why, for example, 30-40 responses to many of the TDAs is enough to report on a "representative sample's" familiarity? (Note, this is not a critique, just a request for an open declaration of why the authors would argue this is response number of sufficient data for these to be usable by others).

I have no other outstanding comments, other than to just state how impressed I am with the transparency of this text and resource. I also find the project highly important and can see many benefits to the field for this work to be shared.

Signed,

Dr Liam Satchell,

Senior Lecturer in Psychology

University of Winchester, UK

liam.satchell@winchester.ac.uk

Journal of Open Psychology Data

http://openpsychologydata.metajnl.com 